\title{
Energy allocation for green multiple relay cooperative communication
}

\author{
Sarmad Sohaib ${ }^{1 *}$ and Daniel KC So ${ }^{2}$
}

\begin{abstract}
Cooperative communication achieves diversity through spatially separated cooperating nodes, which are battery powered in most applications. Therefore the energy consumption must be minimized without compromising the quality of service. In this context, we present a novel energy allocation scheme for multiple relay nodes that results in efficient cooperative multiple-input multiple-output (MIMO) communication. Considering channel path loss, the total transmission energy is distributed between the source and the relay nodes. The energy distribution ratio between the relay and direct link is optimized such that the quality of received signal is maintained with minimum total transmission energy consumption. We calculate the energy distribution ratio analytically and verified it through computer simulation. With the new energy allocation scheme, the system also obtains an increased channel capacity as compared to the cooperative scheme with conventional equal energy allocation and the non-cooperative scheme. Optimal relay positioning with the proposed energy allocation scheme is also explored to maximize the capacity.
\end{abstract}

Keywords: Energy allocation, Cooperative diversity networks, Relay selection, Path loss, Fading channel, Energy efficient communication

\section{Introduction}

The ever increasing demands of spectrum efficiency and link reliability are challenged by the impairments in wireless channel as well as resource constraints. These challenges can be overcome by the use of multiple antennas at the transmitter and receiver, commonly known as multiple-input multiple-output (MIMO) scheme. MIMO systems provide the receiver with multiple versions of an information bearing signal that are subject to independent fading realizations. Thus the multiple spatial channels can be capitalized to provide diversity gain [1-3].

Spatial diversity schemes provide replicas of the transmitted signal through physically separated antennas. Cooperative diversity is an extension of spatial diversity that exploits the broadcast nature of wireless channel. It mimics the performance advantages of MIMO systems and is achieved by the transmission through additional relay nodes [4-12]. The source node broadcasts data to the relay and the destination nodes. The relay node subsequently forwards the received signal to the destination.

\footnotetext{
*Correspondence: sarmad.sohaib@uettaxila.edu.pk

1 Electrical Engineering Department, University of Engineering and

Technology, Taxila, Pakistan

Full list of author information is available at the end of the article
}

Both the direct and relayed signals are combined at the destination using diversity combiners [13].

A relay channel model was introduced and studied by van der Meulen in [14] where he considered communication using three terminals. Cover and El Gamal [10] discussed the discrete memory-less and additive white Gaussian noise relay channel and established the capacity of physically degraded relay channels. Laneman et al. [4] developed several half duplex cooperative protocols including fixed and adaptive relaying protocols. In fixed relay protocols such as amplify-and-forward (ANF) and decode-and-forward (DNF), the relay always assists by forwarding the information from source to destination node. In the case of ANF, the relay simply amplifies the noisy version of the signal transmitted by the source. Whereas in DNF, the relay decodes, re-encodes and retransmits the signals to the destination node. Due to the always forwarding nature for fixed relaying protocol, erroneous data may propagate and cause performance degradation. To overcome this issue, adaptive relaying including selection and incremental relaying is introduced in [4]. With selection relaying, the relay only forwards the information signal if the measured channel amplitude of source-relay (S-R) link lies above a particular threshold.

\section{Springer}

(c) 2012 Sohaib and So; licensee Springer. This is an Open Access article distributed under the terms of the Creative Commons Attribution License (http://creativecommons.org/licenses/by/2.0), which permits unrestricted use, distribution, and reproduction in any medium, provided the original work is properly cited. 
In incremental relaying, limited feedback from the destination is employed to indicate the success and failure of direct transmission. Sendonaris et al. [6,7] examined the detect-and-forward cooperative protocol and gave the theoretical insight of cooperative communication network. They also discussed practical implementation of cooperation for code-division multiple-access systems in which pairs of nodes cooperate with each other and alleviate multiple access interference by using orthogonal spreading codes [6,7]. Hunter and Nosratinia introduced the coded cooperation, where cooperation is achieved through channel coding [8].

Most of the energy constrained networks such as cooperative wireless sensor networks are usually powered by batteries which have limited energy and are mostly difficult to change or recharge [15]. Thus minimizing the energy consumption to maximize the life-time of cooperative network is one of the important factors in network design. Energy efficient protocols must be derived and used for data transfer to increase the network life-time.

In $[16,17]$, it was shown that the total energy consumption in transmission of data can be reduced by using cooperative communication. In $[4,5]$, equal distribution of signal energy between direct and relayed link was used for cooperative communication which is generally not an optimum energy allocation. In [18], the energy allocation expression is derived to maximize the channel capacity in fading environment. In [19], symbol error rate is minimized for optimal energy allocation. However, the extension of these studies to multiple relays network is not obvious. Optimal power allocation in multi-hop network was discussed in [20] where the relay nodes are used for coverage area extension, rather than to provide diversity gain.

In this article, we propose a energy allocation scheme for multi-relay nodes cooperative communication with channel path loss. The scheme aims to minimize the total transmission energy consumption without affecting the link quality. The energy distribution ratio between direct and relayed link $(\Omega)$ is optimized to achieve this aim. This energy distribution ratio is evaluated analytically and is compared with simulation results. The channel capacity with the new energy allocation scheme is also compared with conventional equal energy cooperative scheme and non-cooperative single-input single-output scheme (SISO). In addition, the relay nodes positioning that maximizes the capacity using the proposed energy allocation scheme is also discussed.

The rest of this article is structured as follows. System model and assumptions for cooperative relay channels are introduced in Section '2'. Energy allocation for efficient cooperative communication protocol is explained in Section '3'. Section '4' provides the analytical solution to find the optimal $\Omega$ that minimizes the total transmitted energy consumption. Simulation results demonstrating the marked energy saving in single and two relays case are presented in Section '5'. Capacity analysis is discussed in Section '6.' Concluding remarks are drawn in Section '7'.

\section{System model}

Figure 1 illustrates a $M$-relay wireless network, where data is to be transmitted from source $(S)$ to the destination $(D)$. Due to the broadcast nature of wireless communication, the relay nodes $\left(R_{m}\right)$ will overhear the transmitted information and thus can cooperate with the source to send its data. The conventional ANF channel model is characterized by transmitting and receiving in orthogonal frequency bands or time slots [4]. In this article, we consider the ANF scheme with the relay node transmitting at the same frequency band as the source node, but in subsequent time-slot.

The channel $\tilde{h}_{i j}$ between the $i$ th transmit and $j$ th receive antenna is given by

$$
\tilde{h}_{i j}=\frac{h_{i j}}{\sqrt{P L_{i j}}}
$$

where $h_{i j}$ is the normalized channel gain, which is an independent and identically distributed (i.i.d.) complex Gaussian random variable with zero mean and unit variance. This describes the random fading effect of multipath channels, and is assumed to be quasi-static Rayleigh flat fading. The path loss factor $P L_{i j}$ models the signal attenuation over distance, and is given by [21]

$$
P L_{i j}=\frac{(4 \pi)^{2}}{G_{t} G_{r} \lambda^{2}}\left(d_{i j}\right)^{\alpha}=P L_{0}\left(d_{i j}\right)^{\alpha}
$$

where $P L_{0}$ is the reference path loss factor, $d_{i j}$ is the distance between the $i$ th transmitter and $j$ th receiver, $\alpha$ is the path loss exponent depending on the propagation environment which is assumed to be the same over all links, $\lambda$ is the wavelength, and $G_{t}$ and $G_{r}$ are the transmitter and receiver antenna gains, respectively.

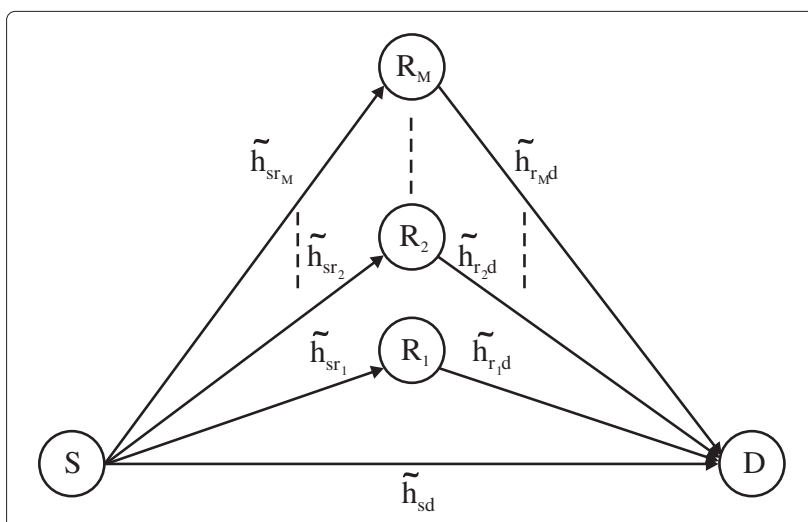

Figure 1 Multi relay cooperative communication network. 
In the first time slot, the source node broadcasts the signal to the destination and the relay node. The received signal at the destination node directly from the source node is

$$
y_{s d}(t)=x(t) \sqrt{\frac{E^{s}}{P L_{s d}}} h_{s d}+n_{d}(t)
$$

where $x(t)$ is the transmitted signal from the source node at time $t$ with energy $E^{s}, h_{s d}$ is the normalized channel gain from the source to the destination node with a corresponding path loss of $P L_{s d}$, and $n_{d}(t)$ captures the effect of additive white Gaussian noise (AWGN) at the destination.

Similarly, at the same time slot the $m$ th relay node receives the same signal from the source node, given by

$$
y_{s r_{m}}(t)=x(t) \sqrt{\frac{E^{s}}{P L_{s r_{m}}}} h_{s r_{m}}+n_{r_{m}}(t)
$$

where $h_{s r_{m}}$ is the normalized channel gain from the source to the $m$ th relay node with a corresponding path loss of $P L_{s r_{m}}$, and $n_{r_{m}}(t)$ is the AWGN.

In the $m$ th time slot, the $m$ th relay node will amplify the received signal from the source node and forwards it to the destination node

$$
y_{r_{m} d}(t+m T)=\sqrt{\frac{k_{m}}{P L_{r_{m} d}}} y_{s r_{m}}(t) h_{r_{m} d}+n_{d}(t+m T)
$$

where $T=L T_{s}$ is the time slot or frame duration with $L$ being the total number of symbols per frame and $T_{s}$ the symbol period, $h_{r_{m} d}$ is the normalized channel gain from the $m$ th relay to destination node having a corresponding path loss of $P L_{r_{m} d}, n_{d}(t+m T)$ is the AWGN at the destination node, and $k_{m}$ is the amplification factor at the $m$ th relay that is used to remain within its power constraints. All AWGN noises are modeled as zero mean independent complex Gaussian random sequences with power spectral density (PSD) $N_{0}$. Exact channel state information (CSI) is assumed to be available at the receiver only, and not at the transmitter. On the other hand, the transmitter is assumed to perfectly estimate the path loss through the reverse link. As the relay nodes are usually spatially well separated, the channel gains for different links are assumed to be independent.

For conventional ANF system, the received signal in (3) and (5) are combined at the destination node using diversity combiners, e.g., Maximal Ratio Combiner (MRC). The diversity gain achieved through cooperation can compensate the additional noise in the relay [2]. Hence, cooperative diversity schemes achieve better performance than non-cooperative schemes.

\section{Cooperative communication with energy allocation}

In conventional ANF, the transmission energy is equally distributed between the direct and the relayed link. This can lead to high energy consumption to achieve a particular performance. By varying the energy ratio between the direct and relayed link, a considerable amount of energy can be saved without affecting the quality of transmission. Thus the network life-time can be considerably increased.

In this section, we analyze the optimum energy allocation for efficient cooperative communication protocol in wireless network with ANF cooperative protocol. We define the energy distribution factor $\Omega_{m}$ as the ratio of received energy at the destination node from the source through the $m$ th relay to the energy received at the destination through the direct link. Mathematically, it can be written as

$$
\begin{aligned}
\Omega_{m} & =\frac{R x \text { Signal Average Energy }\left(S-R_{m}-D\right)}{R x \text { Signal Average Energy }(S-D)} \\
& =\frac{\left(k_{m} \mathbb{E}\left[\left|h_{r_{m} d}\right|^{2}\right] E^{r_{m}}\right) / P L_{r_{m} d}}{\mathbb{E}\left[\left|h_{s d}\right|^{2}\right] E^{s} / P L_{s d}} \\
& =\frac{\left(k_{m} \mathbb{E}\left[\left|h_{r_{m} d}\right|^{2}\right] / P L_{r_{m} d}\right)\left(\mathbb{E}\left[\left|h_{s r_{m}}\right|^{2}\right] E^{s} / P L_{s r_{m}}+N_{0}\right)}{\mathbb{E}\left[\left|h_{s d}\right|^{2}\right] E^{s} / P L_{s d}}
\end{aligned}
$$

where $E^{r_{m}}$ is the transmitted signal energy from the $m$ th relay node. The channels $h_{i j}$ between the $i$ th transmitter and $j$ th receiver are assumed Rayleigh faded, therefore $\mathbb{E}\left[\left|h_{i j}\right|^{2}\right]=1$, and (6) becomes

$$
\Omega_{m}=\frac{k_{m}}{E^{s}}\left(\frac{d_{s d}}{d_{r_{m} d}}\right)^{\alpha}\left(\frac{E^{s}}{P L_{s r_{m}}}+N_{0}\right)
$$

For realistic bit error rates, the required SNR will have $E^{s} / P L_{s r_{m}}$ much larger than $N_{0}$. Thus, neglecting $N_{0}$ in (7), $\Omega_{m}$ can be simplified to

$$
\Omega_{m}=\frac{k_{m}}{P L_{0}}\left(\frac{d_{s d}}{d_{s r_{m}} d_{r_{m} d}}\right)^{\alpha} .
$$

If $\Omega_{m}$ is small, most of the signal energy will flow directly to the destination from the source node. In other words, higher transmission energy is allocated to the source node than the relay node(s). On the other hand, when $\Omega_{m}$ is high, a large amount of energy flows to destination through the relay link than the direct one. Substituting $y_{s r_{m}}(t)$ in (4) and $k_{m}$ in (8) into (5), we get

$$
\begin{aligned}
y_{r_{m} d}(t+m T)= & \sqrt{\frac{\Omega_{m}}{P L_{s d}} E^{s}} x(t) h_{s r_{m}} h_{r_{m} d} \\
& +\sqrt{\Omega_{m}\left(\frac{d_{s r_{m}}}{d_{s d}}\right)^{\alpha}} h_{r_{m} d} n_{r_{m}}(t)+n_{d}(t+m T) .
\end{aligned}
$$


MRC is employed at the destination node where $M+$ 1 received signals, directly from the source node and through the $M$-relay nodes are combined. The received SNR of the direct link from the source node at destination is given as

$$
\gamma_{s d}=\frac{E^{s}}{P L_{s d} N_{0}}
$$

The received SNR at the $m$ th-relay from the source node is

$$
\gamma_{s r_{m}}=\frac{E^{S}}{P L_{s r_{m}} N_{0}}
$$

and the received SNR of the $m$ th-relay to the destination link becomes

$$
\gamma_{r_{m} d}=\frac{E^{s}}{P L_{s d} N_{0}}\left(\frac{\Omega_{m}}{1+\Omega_{m}\left(\frac{d_{s r_{m}}}{d_{s d}}\right)^{\alpha}}\right) .
$$

The total received SNR at the destination node after MRC is given by

$$
\begin{aligned}
& \gamma_{\text {total }}=\gamma_{s d}+\sum_{m=1}^{M} \gamma_{r_{m} d} \\
& =\frac{E^{s}}{P L_{s d} N_{0}}\left(1+\sum_{m=1}^{M} \frac{\Omega_{m}}{1+\Omega_{m}\left(\frac{d_{s r_{m}}}{d_{s d}}\right)^{\alpha}}\right) .
\end{aligned}
$$

The total transmitted signal energy $\left(E_{T}\right)$ is defined as the sum of transmitted energy from the source and relay nodes and is given by

$$
\begin{aligned}
E_{T} & =E^{S}+\sum_{m=1}^{M} E^{r_{m}}=E^{s}+E^{s} \sum_{m=1}^{M} \frac{k_{m}}{P L_{s r_{m}}} \\
& =E^{s}\left(1+\sum_{m=1}^{M} \Omega_{m}\left(\frac{d_{r_{m} d}}{d_{s d}}\right)^{\alpha}\right) .
\end{aligned}
$$

\section{Optimal energy distribution factor for multi-relay network}

In the previous section, we presented the signal model for cooperative communication with energy allocation. In this section, the optimal energy distribution factor that minimizes the total transmitted energy consumption will be determined analytically. Assuming the channels $h_{i j}$ are Rayleigh faded, the average probability of error at high SNR region is given by [22]

$$
P_{e} \approx W(M) \frac{1}{\gamma_{s d}} \prod_{m=1}^{M}\left(\frac{1}{\gamma_{s r_{m}}}+\frac{1}{\gamma_{r_{m} d}}\right)
$$

where

$$
W(M)=\frac{(2)^{M+1} \prod_{u=1}^{M+1}(2 u-1)}{2(M+1) ! l^{(2 M+2)}}
$$

and $l$ depends upon the type of modulation ( 2 for phase shift keying (PSK)). It clearly shows that by using $M$ relays in the cooperative network, a diversity order of $M+1$ is achieved. Substituting the values of $\gamma_{s d}, \gamma_{s r_{m}}$ and $\gamma_{r_{m} d}$ from (10), (11), and (12), respectively, into (16),

$$
\begin{aligned}
P_{e}= & W(M)\left(\frac{P L_{s d} N_{0}}{E^{s}}\right) \prod_{m=1}^{M} \\
& \times\left(\frac{P L_{s r_{m}} N_{0}}{E^{s}}+\frac{P L_{s d} N_{0}}{E^{s}}\left(\frac{1+\Omega_{m}\left(d_{s r_{m}} / d_{s} d\right)^{\alpha}}{\Omega_{m}}\right)\right) \\
= & W(M)\left(\frac{P L_{s d} N_{0}}{E^{s}}\right)^{M+1} \prod_{m=1}^{M} \\
& \times\left(\left(\frac{d_{s r_{m}}}{d_{s d}}\right)^{\alpha}+\frac{1+\Omega_{m}\left(d_{s r_{m}} / d_{s d}\right)^{\alpha}}{\Omega_{m}}\right) \\
= & W(M)\left(\frac{P L_{s d} N_{0}}{E^{s}}\right)^{M+1} \prod_{m=1}^{M}\left(\frac{1}{\Omega_{m}}+2\left(\frac{d_{s r_{m}}}{d_{s d}}\right)^{\alpha}\right) .
\end{aligned}
$$

Rearranging (18), $E^{S}$ becomes

$$
E^{s}=P L_{s d} N_{0}\left[\frac{W(M)}{P_{e}} \prod_{m=1}^{M}\left(\frac{1}{\Omega_{m}}+2\left(\frac{d_{s r_{m}}}{d_{s d}}\right)^{\alpha}\right)\right]^{1 /(M+1)} .
$$

Substituting the value of $E^{S}$ into (15), we obtain the total transmitted energy to be

$$
\begin{aligned}
E_{T}= & P L_{s d} N_{0}\left(1+\sum_{m=1}^{M} \Omega_{m}\left(\frac{d_{r_{m} d}}{d_{s d}}\right)^{\alpha}\right) \\
& \times\left[\frac{W(M)}{P_{e}} \prod_{m=1}^{M}\left(2\left(\frac{d_{s r_{m}}}{d_{s d}}\right)^{\alpha}+\frac{1}{\Omega_{m}}\right)\right]^{1 /(M+1)} .
\end{aligned}
$$

Assuming

$$
g\left(\Omega_{m}\right)=\sum_{m=1}^{M} \Omega_{m}\left(\frac{d_{r_{m} d}}{d_{s d}}\right)^{\alpha}
$$

and

$$
f\left(\Omega_{m}\right)=\prod_{m=1}^{M}\left(2\left(\frac{d_{s r_{m}}}{d_{s d}}\right)^{\alpha}+\frac{1}{\Omega_{m}}\right),
$$

Equation (20) becomes

$$
E_{T}=P L_{s d} N_{0}\left(1+g\left(\Omega_{m}\right)\right)\left[\frac{W(M)}{P_{e}} f\left(\Omega_{m}\right)\right]^{1 /(M+1)} .
$$


To minimize $E_{T}$ with respect to (w.r.t.) $\Omega_{m}$, we take the derivative of (23) w.r.t. $\Omega_{m}$ and set it to zero

$$
\frac{d E_{T}}{d \Omega_{m}}=0
$$

Solving (24), we get

$$
\begin{aligned}
& \quad\left(f\left(\Omega_{m}\right)\right)^{1 /(M+1)} \frac{d}{d \Omega_{m}} g\left(\Omega_{m}\right)+\frac{d}{d \Omega_{m}} f\left(\Omega_{m}\right) \frac{1}{M+1} \\
& \times f\left(\Omega_{m}\right)^{-M /(M+1)}\left(1+g\left(\Omega_{m}\right)\right)=0 .
\end{aligned}
$$

Thus, using (25), the optimal values of $\Omega_{m}$ for any number of relay nodes can be calculated. In the following sections we present the calculations for one and two relay nodes only.

\subsection{Solving for one relay node}

For the ease of notation we have dropped subscript 1 from the $\Omega$ in the following equations. For one relay i.e. $m=1$, (25) becomes

$$
\begin{aligned}
& -\frac{1}{2 \Omega^{2}}\left(\frac{1}{\Omega}+2\left(\frac{d_{s r}}{d_{s d}}\right)^{\alpha}\right)^{-1 / 2}\left(1+\Omega\left(\frac{d_{r d}}{d_{s d}}\right)^{\alpha}\right) \\
& +\left(\frac{d_{r d}}{d_{s d}}\right)^{\alpha} \sqrt{\frac{1}{\Omega}+2\left(\frac{d_{s r}}{d_{s d}}\right)^{\alpha}}=0 \\
& \Rightarrow 4\left(\frac{d_{s r}}{d_{s d}}\right)^{\alpha} \Omega^{2}+\Omega-\left(\frac{d_{s d}}{d_{r d}}\right)^{\alpha}=0 .
\end{aligned}
$$

Solving (27), we have

$$
\Omega=\frac{1}{8}\left(\frac{d_{s r}}{d_{s d}}\right)^{-\alpha}\left(-1+\sqrt{1+16\left(\frac{d_{s r}}{d_{r d}}\right)^{\alpha}}\right) .
$$

The above expression can be used to find the optimal energy distribution between the source and the single relay node.

\subsection{Solving for two relay nodes}

In this section, we calculate the optimal energy allocation for two relays cooperative communication network. For $M=2$, solving (25) with $m=\{1,2\}$, we obtain

$$
\begin{gathered}
\left(\frac{d_{r_{1} d}}{d_{s d}}\right)^{\alpha}\left(2\left(\frac{d_{s r_{1}}}{d_{s d}}\right)^{\alpha}+\frac{1}{\Omega_{1}}\right)-\frac{1}{3 \Omega_{1}^{2}} \\
\times\left(1+\left(\frac{d_{r_{1} d}}{d_{s d}}\right)^{\alpha} \Omega_{1}+\left(\frac{d_{r_{2} d}}{d_{s d}}\right)^{\alpha} \Omega_{2}\right)=0
\end{gathered}
$$

and

$$
\begin{gathered}
\left(\frac{d_{r_{2} d}}{d_{s d}}\right)^{\alpha}\left(2\left(\frac{d_{s r_{2}}}{d_{s d}}\right)^{\alpha}+\frac{1}{\Omega_{2}}\right)-\frac{1}{3 \Omega_{2}^{2}} \\
\times\left(1+\left(\frac{d_{r_{1} d}}{d_{s d}}\right)^{\alpha} \Omega_{1}+\left(\frac{d_{r_{2} d}}{d_{s d}}\right)^{\alpha} \Omega_{2}\right)=0 .
\end{gathered}
$$

Equation (29) and (30) can be simplified to quadratic form

$$
2\left(\frac{d_{s r_{1}} d_{r_{1} d}}{d_{s d}^{2}}\right)^{\alpha} \Omega_{1}^{2}+\frac{2}{3}\left(\frac{d_{r_{1} d}}{d_{s d}}\right)^{\alpha} \Omega_{1}-\frac{1}{3}\left(1+\left(\frac{d_{r_{2} d}}{d_{s d}}\right)^{\alpha} \Omega_{2}\right)=0
$$

and

$2\left(\frac{d_{s r_{2}} d_{r_{2} d}}{d_{s d}^{2}}\right)^{\alpha} \Omega_{2}^{2}+\frac{2}{3}\left(\frac{d_{r_{2} d}}{d_{s d}}\right)^{\alpha} \Omega_{2}-\frac{1}{3}\left(1+\left(\frac{d_{r_{1} d}}{d_{s d}}\right)^{\alpha} \Omega_{1}\right)=0$.

Now solving the (31) for $\Omega_{1}$ and (32) for $\Omega_{2}$, we have

$\Omega_{1}=\frac{1}{6}\left(\frac{d_{s r_{1}}}{d_{s d}}\right)^{-\alpha}\left(-1+\sqrt{1+6\left(\frac{d_{s r_{1}}}{d_{r_{1} d}}\right)^{\alpha}+6\left(\frac{d_{s r_{1}} d_{r_{2} d}}{d_{r_{1} d} d_{s d}}\right)^{\alpha} \Omega_{2}}\right)$

and

$\Omega_{2}=\frac{1}{6}\left(\frac{d_{s r_{2}}}{d_{s d}}\right)^{-\alpha}\left(-1+\sqrt{1+6\left(\frac{d_{s r_{2}}}{d_{r_{2} d}}\right)^{\alpha}+6\left(\frac{d_{s r_{2}} d_{r_{1} d}}{d_{r_{2} d} d_{s d}}\right)^{\alpha} \Omega_{1}}\right)$.

The above expressions can be used to calculate the optimal distribution ratios $\Omega_{1}$ and $\Omega_{2}$. As (33) and (34) are interlinked, they are solved iteratively to find the values of $\Omega_{1}$ and $\Omega_{2}$.

\subsection{Solving for three relay nodes}

The optimal energy allocation for three relays cooperative communication network is presented in this section. For $M=3$, solving (25) with $m=\{1,2,3\}$, for $\Omega_{1}, \Omega_{2}$, and $\Omega_{3}$ we obtain 


$$
\begin{aligned}
& \Omega_{1}=\frac{1}{16}\left(\frac{d_{s r_{1}}}{d_{s d}}\right)^{-\alpha}\left(-3+\sqrt{9\left(\frac{d_{r_{1} d}}{d_{s d}}\right)^{\alpha}+32\left(\frac{d_{s r_{1}}}{d_{s d}}\right)^{\alpha}\left(1+\left(\frac{d_{r_{2} d}}{d_{s d}}\right)^{\alpha} \Omega_{2}+\left(\frac{d_{r_{3} d}}{d_{s d}}\right)^{\alpha} \Omega_{3}\right)}\right), \\
& \Omega_{2}=\frac{1}{16}\left(\frac{d_{s r_{2}}}{d_{s d}}\right)^{-\alpha}\left(-3+\sqrt{9\left(\frac{d_{r_{2} d}}{d_{s d}}\right)^{\alpha}+32\left(\frac{d_{s r_{2}}}{d_{s d}}\right)^{\alpha}\left(1+\left(\frac{d_{r_{1} d}}{d_{s d}}\right)^{\alpha} \Omega_{1}+\left(\frac{d_{r_{3} d}}{d_{s d}}\right)^{\alpha} \Omega_{3}\right)}\right)
\end{aligned}
$$

and

$$
\Omega_{3}=\frac{1}{16}\left(\frac{d_{s r_{3}}}{d_{s d}}\right)^{-\alpha}\left(-3+\sqrt{9\left(\frac{d_{r_{3} d}}{d_{s d}}\right)^{\alpha}+32\left(\frac{d_{s r_{3}}}{d_{s d}}\right)^{\alpha}\left(1+\left(\frac{d_{r_{1} d}}{d_{s d}}\right)^{\alpha} \Omega_{1}+\left(\frac{d_{r_{2} d}}{d_{s d}}\right)^{\alpha} \Omega_{2}\right)}\right) .
$$

Iteratively solving the above expressions will give the optimal distribution ratios $\Omega_{1}, \Omega_{2}$, and $\Omega_{3}$.

Therefore using (25) the optimal value of $\Omega_{m}$ is calculated for any number of relay nodes that is further used to adjust the amplification factor $k_{m}$, hence adjust the source and relay transmit power. Although the generic path loss model is considered in this article, it can easily be extended to any realistic model that is proportional to distance with arbitrary exponent (i.e., $\propto d^{\alpha}$ ), such as the COST 231 model [23].

\section{Simulation results and analysis}

In this section, we present the analytical results we derived in Section '4', and validate it using simulation results. QPSK modulation scheme is used in the analysis and simulation. To obtain reasonable values of SNR, the transmitted signal from source node is amplified by $\sqrt{P L_{s d}}$ to compensate for the path loss. The carrier frequency $f_{c}$ is set to be $2.5 \mathrm{GHz}$. The path loss exponent $\alpha$ is taken to be 3.5. The $G_{t} G_{r}$ and $N_{0} / 2$ is set to be $5 \mathrm{dBi}$ and $-174 \mathrm{dBm} / \mathrm{Hz}$, respectively. For all simulation results the total distance from the source to the relay and to the destination is kept constant. It is also set to be equal to the distance from the source to the destination node, i.e., $d_{s r_{m}}+d_{r_{m} d}=d_{s d}=200 \mathrm{~m}$. The total energy consumption is calculated using (15), where $E^{s}$ is obtained using total received SNR $\gamma_{\text {total }}$ in (13) observed at BER $=10^{-3}$. The total received SNR is obtained by evaluating the BER over 10,000 randomly generated channel samples at each $\Omega$ or transmission distance.

As can be seen from (28), (33), (34), (35), (36), and (37) the optimal energy distribution factor is a function of distances between the nodes which are assumed to be known at all the relay and source nodes.

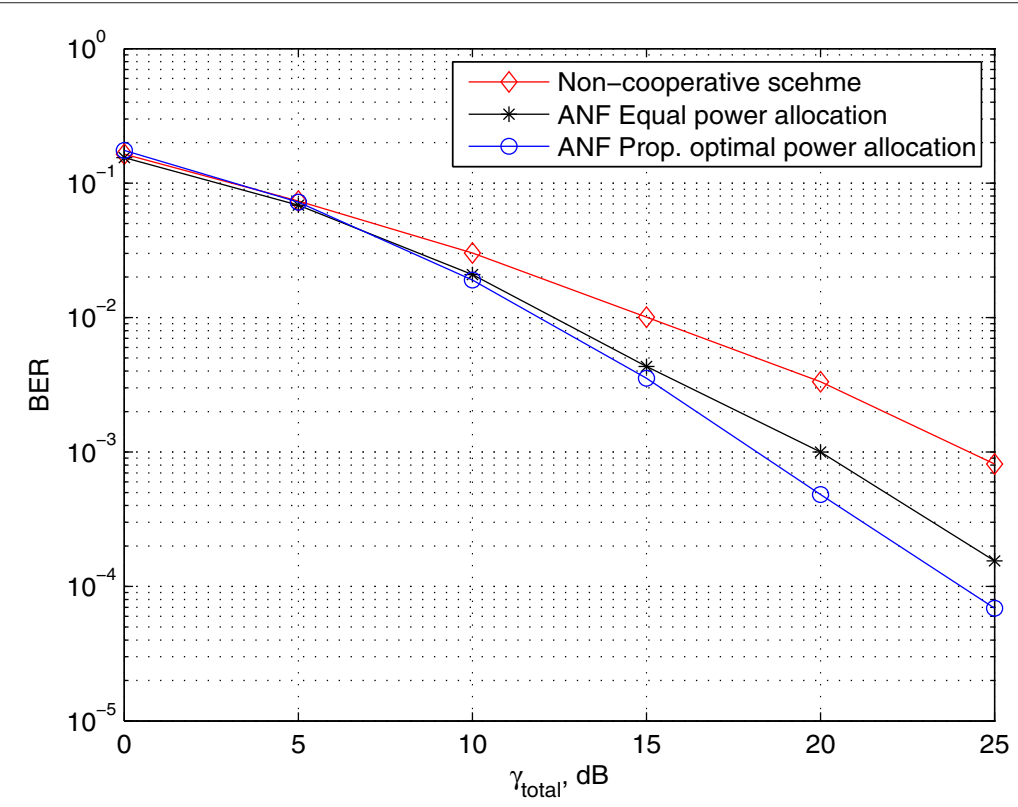

Figure 2 BER for single relay system as a function of $\gamma_{\text {total }}$. 


\subsection{Single relay scenario}

In this section, the simulation results obtained from the single relay cooperative network are discussed. Unless and until stated, the relay is always assumed to be located midway between the source and the destination. Solving (28) with the defined parameters, the value of $\Omega$ is found to be 4.4.

Figure 2 shows the BER comparison of non-cooperative scheme, ANF with equal power allocation and ANF with proposed power allocation scheme as a function of $\gamma_{\text {total }}$. It can be observed that the total SNR required by the proposed scheme to achieve a certain BER is less than the equal power allocation scheme. This is because the proposed scheme minimizes the total transmitted energy according to a BER function. Thus, the proposed scheme will require a lower SNR to achieve a target BER, and thus the improved performance.

The simulated and analytical results of the total transmitted energy as a function of $\Omega$ at $\mathrm{BER}=10^{-3}$ are presented in Figure 3. We compare the two results and show that the two sets of values fit well. Analytically, we obtain a minimum $E_{T}$ at $\Omega=4.4$, whereas it is approximately 6 in the simulated results. The difference between them could be due to the assumptions made in the probability of error in (16) [22]. Nonetheless, the total transmitted energy is close to the empirical result with only $3.3 \%$ difference at $\Omega=4.4$.

Figure 4 shows the total transmission energy consumption for different relay positions. The results shows that the proposed energy allocation scheme is more energy efficient than both the equal energy allocation scheme and energy allocation scheme of [19]. It can be observed that the proposed scheme has high energy consumption if the relay is placed near the source and decreases as the relay moves away from it. The minimum total transmitted energy is observed when the relay is approximately $125 \mathrm{~m}$ away from the source node, and after which the total energy consumption again increases as the relay moves towards the destination node. The minimum energy consumption is not obtained exactly at the middle, which can be explained using (15). The minimum simulated $E^{S}$ is achieved when the relay is located at the midpoint between source and destination node. However the multiplicative factor $\left(1+\left(d_{r d} / d_{s d}\right)^{\alpha}\right)$ decreases as the relay moves away from the source node and therefore it shifts the minimum $E_{T}$ away from the center. Another observation is the higher energy consumption when the relay is positioned nearer the source than when it is closer to the destination. Again (15) can be used to explain this trend. When the relay moves nearer the destination, $d_{r d} / d_{s d}$ decreases and $E_{T}$ tends to $E^{S}$. On the contrary, when the relay is located near the source, $d_{r d} / d_{s d}$ becomes unity meaning $E_{T}$ approaches $2 E^{S}$. Although the value of $E^{S}$ is different in both cases, the difference is much less than the multiplicative factor two that actually increase $E_{T}$ when the relay is located near the source.

This will contribute to optimal design for the lowpower high-efficiency communication system which corresponds to the number of antennas for which we can get the lowest costs in energy consumption for a required level of system capacity.

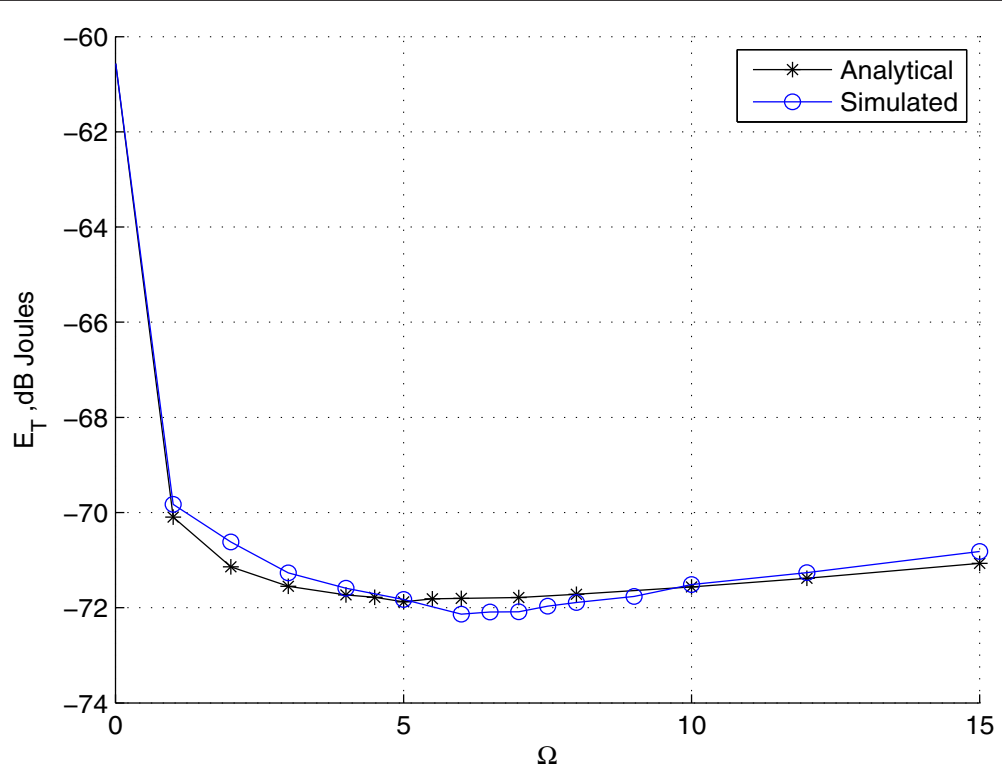

Figure 3 Simulated and analytically calculated $E_{T}$ as a function of $\Omega$ at $\mathrm{BER}=10^{-3}$ for single relay system. 


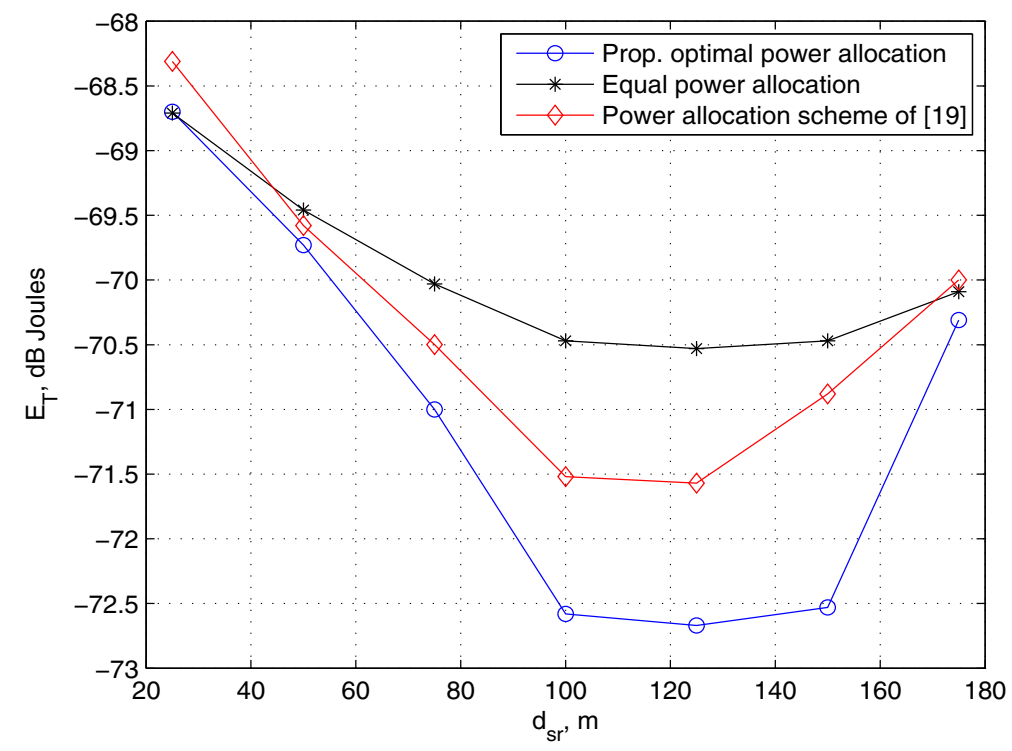

Figure 4 Single relay node total transmission energy consumption for different relay positions at $\mathrm{BER}=10^{-3}$.

\subsection{Two relays scenario}

The total energy consumption comparison among the proposed optimal scheme, equal energy scheme and energy allocation scheme of [19] for two relays system where $d_{s r_{1}}=d_{r_{2} d}$, is shown in Figure 5. The performance of the proposed scheme degrades as one of the relay nodes gets close to the source or destination. The optimal performance is achieved when the relays move to the center between source and destination. It should be noted that, in Figure 5 the optimal power allocation factors, $\Omega_{1}$ and $\Omega_{2}$, for each relay node are different due to the different relay positions.
Different positioning for the two relay nodes have been evaluated and shows that the optimal energy allocation scheme outperforms both the equal allocation scheme and energy allocation scheme of [19]. These results are thus not presented for brevity.

\subsection{Three relays scenario}

Figure 6 illustrates the total energy consumption comparison between the proposed optimal and equal energy scheme. In this simulation, the $\mathrm{R} 1$ is assumed to be located midway between the source and the destination node and $d_{s r_{2}}=d_{r_{3} d}$. It can be observed that the proposed energy

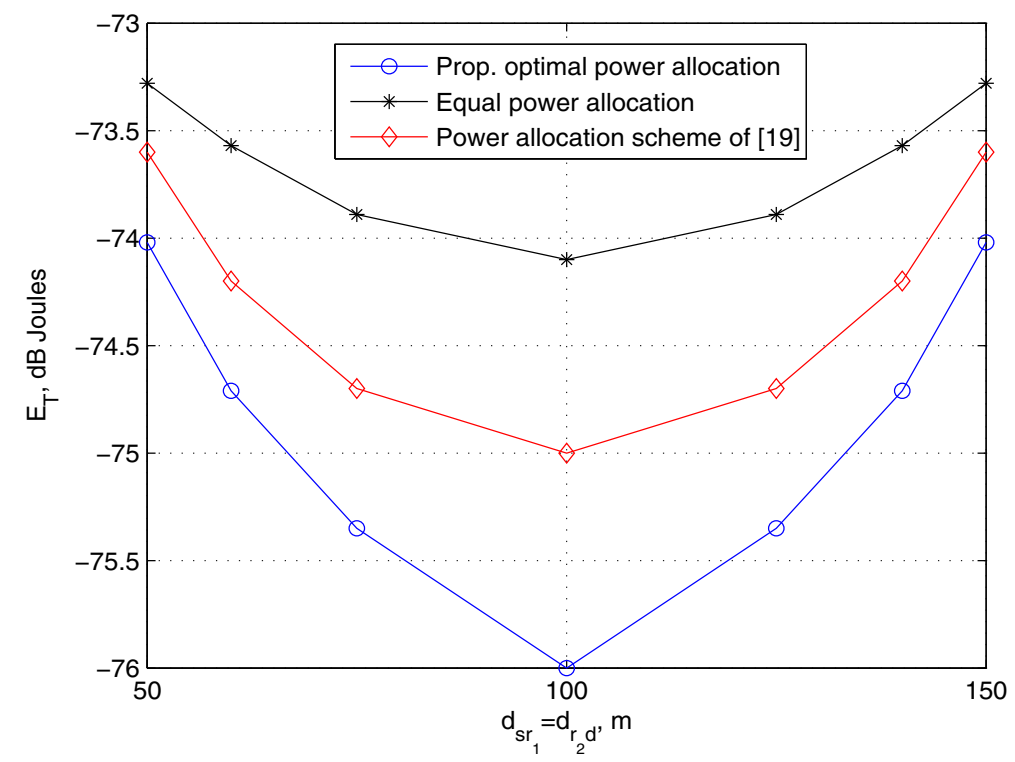

Figure $5 E_{T}$ as a function of $d_{s r_{1}}=d_{r_{2} d}$ at BER $=10^{-3}$ 


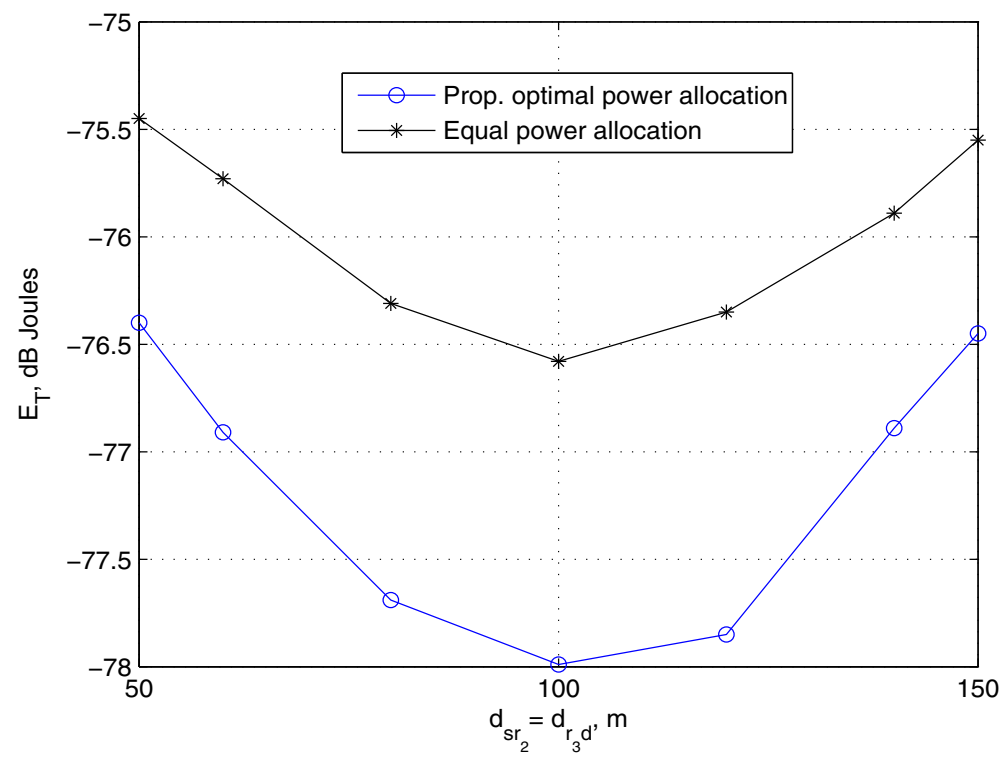

Figure 6 Three relay nodes total transmission energy consumption with one relay in mid point at $B E R=10^{-3}$.

allocation scheme is more energy efficient than the equal energy allocation scheme. The optimal performance is achieved when the relays move to the centre between source and destination.

\section{Capacity analysis}

In this section, the capacity of the proposed energy allocation scheme for $M$-relay cooperative system is presented and compared with conventional equal energy allocation scheme and energy allocation scheme of [19]. Given the channel information at the receiver, the channel capacity of the proposed system with $M$ relays can be calculated as [4]

$$
C=\frac{1}{M+1} \log _{2}\left(1+\gamma_{s d}+\gamma_{s r_{m} d}\right)
$$

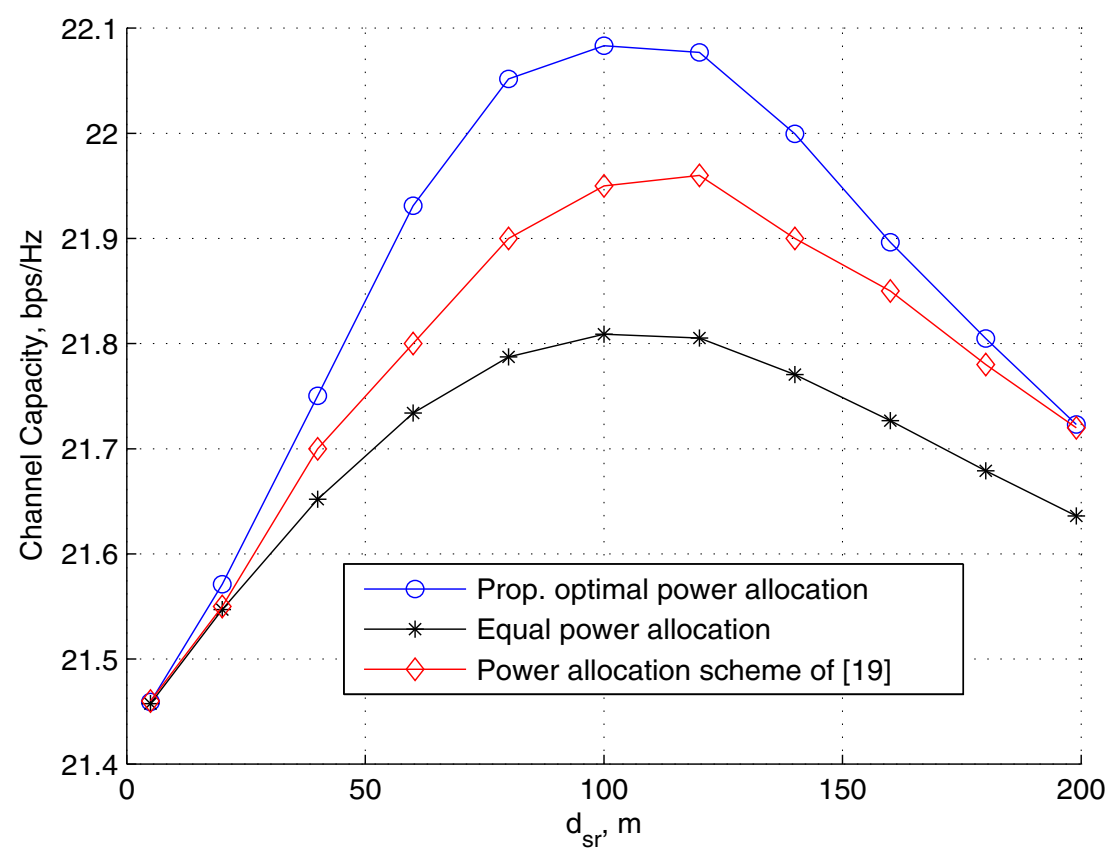

Figure 7 Single relay capacity comparison with different energy allocation schemes at $E_{T}=-72 \mathrm{~dB} \mathrm{~J}$. 


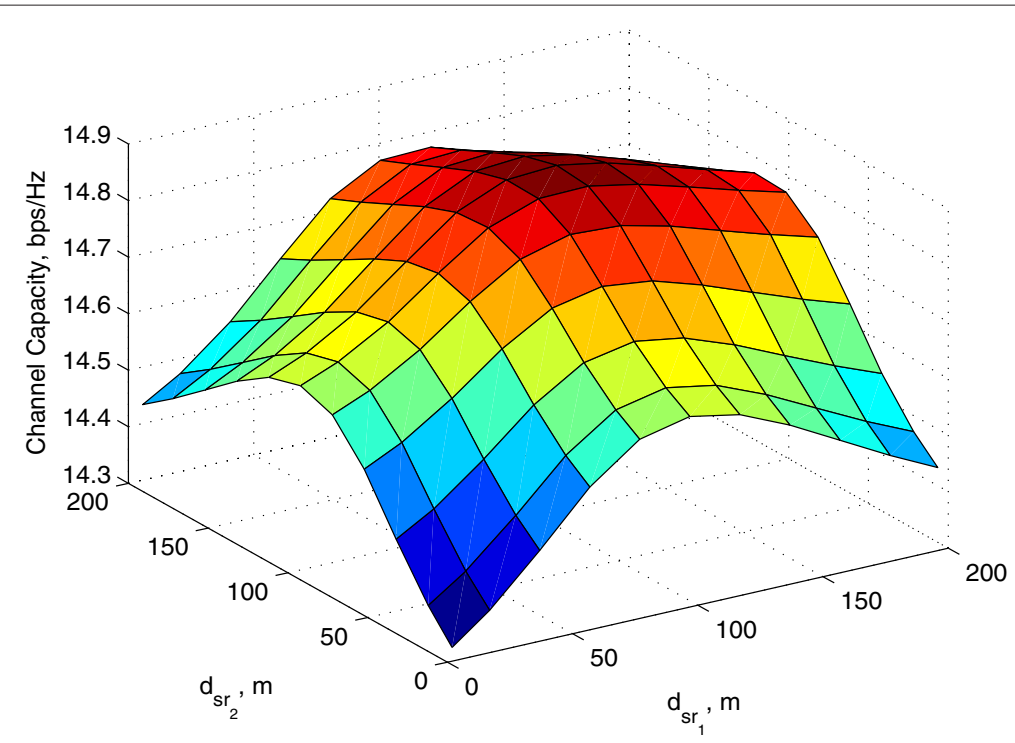

Figure 8 Two relays capacity at different relay positions at $E_{T}=-72 \mathrm{~dB} \mathrm{~J}$.

where

$$
\gamma_{s r_{m} d}=\sum_{m=1}^{M} \frac{\gamma_{s r_{m}} \gamma_{r_{m} d}}{1+\gamma_{s r_{m}}+\gamma_{r_{m} d}}
$$

Figure 7 shows the single relay channel capacity of the proposed energy allocation, the conventional equal energy allocation and the energy allocation scheme in [19] as a function of S-R distance at $E_{T}=-72 \mathrm{dBJ}$. It can be observed that the proposed energy allocation scheme achieves a higher capacity. This is again due to the proposed scheme minimizing the required transmitted energy to achieve a target BER. With the same transmitted energy, the proposed allocation scheme will obtain a higher overall SNR than the other schemes, and thus the improved capacity.

For the two relay case, Figure 8 shows the channel capacity for different relay positions with optimal energy allocation scheme. The maximum capacity is achieved when the relays are at approximately $125 \mathrm{~m}$ away from the source. It should be noted that, in Figure 8 the optimal power allocation factors, $\Omega_{1}$ and $\Omega_{2}$, are different for different relay positions.

\section{Conclusions}

In this article, a novel energy allocation scheme for multiple relay conventional ANF protocol is presented. It aims to minimize the total transmission energy without affecting the error rate performance. Considering channel path loss, the total transmission energy is distributed between the source and the relay nodes. A generalized equation for the optimal energy distribution ratio between the relay and direct link is derived. The proposed solution allows fast computation on the required transmission energy in the source and relay node. The special cases with single, two and three relay nodes are analyzed and is shown to be close to the simulated results. The BER, total energy consumption and capacity analysis confirm the performance improvement over conventional equal energy allocation.

\section{Competing interests}

The authors declare that they have no competing interests.

\section{Author details}

${ }^{1}$ Electrical Engineering Department, University of Engineering and Technology, Taxila, Pakistan. ${ }^{2}$ School of Electrical and Electronics Engineering, University of Manchester, Manchester, UK.

Received: 25 January 2012 Accepted: 29 July 2012

Published: 18 September 2012

\section{References}

1. A Basyouni, W Hamouda, A Youssef, Cooperative relaying protocol for energy-constrained ad hoc networks. IET Commun. 5(4), 418-424 (2011)

2. Y Kim, H Liu, Infrastructure relay transmission with cooperative MIMO. IEEE Trans. Veh. Technol. 57(4), 2180-2188 (2008)

3. H Rutagemwa, TJ Willink, L Li, Modeling and performance analysis of multihop cooperative wireless networks. IEEE Trans. Veh. Technol. 59(6), 3057-3069 (2010)

4. JN Laneman, DNC Tse, GW Wornell, Cooperative diversity in wireless networks: efficient protocols and outage behavior. IEEE Trans. Inf. Theory. 50(12), 3062-3080 (2004)

5. JN Laneman, GW Wornell, Distributed space-time-coded protocols for exploiting cooperative diversity in wireless networks. IEEE Trans. Inf. Theory. 49(10), 2415-2425 (2003)

6. A Sendonaris, E Erkip, B Aazhang, User cooperation diversity, Part I: system description. IEEE Trans. Commun. 51(11), 1927-1938 (2003)

7. A Sendonaris, E Erkip, B Aazhang, User cooperation diversity, Part II: implementation aspects and performance analysis. IEEE Trans. Commun. 51(11), 1939-1948 (2003)

8. TE Hunter, A Nosratinia, Diversity through coded cooperation. IEEE Trans. Wirel. Commun. 5(2), 283-289 (2006)

9. A Nosratinia, TE Hunter, A Hedayat, Cooperative communication in wireless networks. IEEE Commun. Mag. 42(10), 74-80 (2004) 
10. T Cover, AE Gamal, Capacity theorems for the relay channel. IEEE Trans. Inf. Theory. 25(5), 572-584 (1979)

11. S Sohaib, DKC So, in Proc. IEEE 69th Vehicular Technology Conference, 1-5. Asynchronous polarized cooperative MIMO communication, (2009)

12. M Torabi, D Haccoun, W Ajib, Performance analysis of cooperative diversity with relay selection over non-identically distributed links. IET Commun. 4(5), 596-605 (2010)

13. DG Brennan, Linear diversity combining techniques. Proc. IEEE. 91(2), 331-356 (2003)

14. EC van der Meulen, Three-terminal communication channels. Adv. Appl. Prob. 3(1), 120-154 (1971)

15. IF Akyildiz, W Su, Y Sankarasubramaniam, E Cayirci, A survey on sensor networks. IEEE Commun. Mag. 40(8), 102-114 (2002)

16. S Cui, AJ Goldsmith, A Bahai, Energy-efficiency of MIMO and cooperative MIMO techniques in sensor networks. IEEE J. Sel. Areas Commun. 22(6), 1089-1098 (2004)

17. Z Chen, C Yang, in Proc 8th International Conference on Signal Processing, vol. 4. Energy efficiency of cooperative diversity at PHY layer in wireless sensor networks, (2006)

18. Z Jingmei, Z Qi, S Chunju, W Ying, Z Ping, Z Zhang, in IEEE 59th Vehicular Technology Conference, vol. 2. Adaptive optimal transmit power allocation for two-hop non-regenerative wireless relaying system, (2004), pp. 1213-1217

19. W Su, AK Sadek, KJR Liu, Cooperative communication protocols in wireless networks: performance analysis and optimum power allocation. Wirel. Person. Commun. 44(2), 181-217 (2008)

20. MO Hasna, MS Alouini, Optimal power allocation for relayed transmissions over Rayleigh-fading channels. IEEE Trans. Wirel. Commun. 3(6), 1999-2004 (2004)

21. S Haykin, M Moher, Modern Wireless Communications. (Prentice Hall, Upper Saddle River, 2004)

22. A Ribeiro, X Cai, GB Giannakis, Symbol error probabilities for general Cooperative links. IEEE Trans. Wirel. Commun. 4(3), 1264-1273 (2005)

23. AF Molisch, Wireless Communications. (Wiley, New York, 2010)

\section{doi:10.1186/1687-1499-2012-291}

Cite this article as: Sohaib and So: Energy allocation for green multiple relay cooperative communication. EURASIP Journal on Wireless Communications and Networking 2012 2012:291.

\section{Submit your manuscript to a SpringerOpen ${ }^{\circ}$ journal and benefit from:}

- Convenient online submission

- Rigorous peer review

- Immediate publication on acceptance

- Open access: articles freely available online

- High visibility within the field

- Retaining the copyright to your article

Submit your next manuscript at $\boldsymbol{\triangleright}$ springeropen.com 